Mediterránea Ser. Biol. (1985), n. ${ }^{\circ}$ 8. Págs. 5-15

\title{
CHTHONIUS (EPHIPPIOCHTHONIUS) VERAI NUEVA ESPECIE CAVERNICOLA DEL SURESTE ESPAÑOL (ARACHNIDA, PSEUDOSCORPIONES, CHTHONIIDAE)
}

\author{
por \\ Juan Antonio Zaragoza1
}

\section{INTRODUCCION}

La propuesta formulada más abajo para una nueva especie del subgénero Ephippiochthonius, viene a aumentar la lista de endemismos propios del Sureste Español. Nuevamente se pone en evidencia que, la búsqueda sistemática en esta Zona y tanto en biotopos hipogeos o epigeos, puede proporcionar un número aún considerable de nuevas especies.

\section{SUMMARY}

A new cave-dwelling species of Ephippiochthonius from the Southeast of Spain in proposed: Chthonius (E.) verai $\mathrm{n}$. sp. belongs to the group of species with basal lamella on the movable finger of the chela; its relationship with iberian and mediterranean species is discussed.

Chthonius (Ephispiochthonius) verai $\mathrm{n} . \mathrm{sp}$.

Material: Cova del Tío Melxor, Castalla, Alicante; J.A. Zaragoza 1g., 18-VI-78: 1 ơ (Alotipo, col. autor); V. Capdepón/A. Sendra/J.A. Zaragoza 1gs., 07-X-84: 4 \& (Holotipo y 3 Paratipos, col. autor).

Diagnosis: Ejemplar de tamaño relativamente grande para el subgénero. Ojos reducidos a manchas claras o con restos del par anterior. Parcialmente despigmentado. 18 sedas en el prosoma, sin microsedas preoculares. Dedo móvil de la pinza del pedipalpo con lámina basal amplia y ondulada. Tricobotrio ist claramente distal de $e s b$.

(1) Departamento de Biología, Fac. Ciencias, Univ. Alicante. 


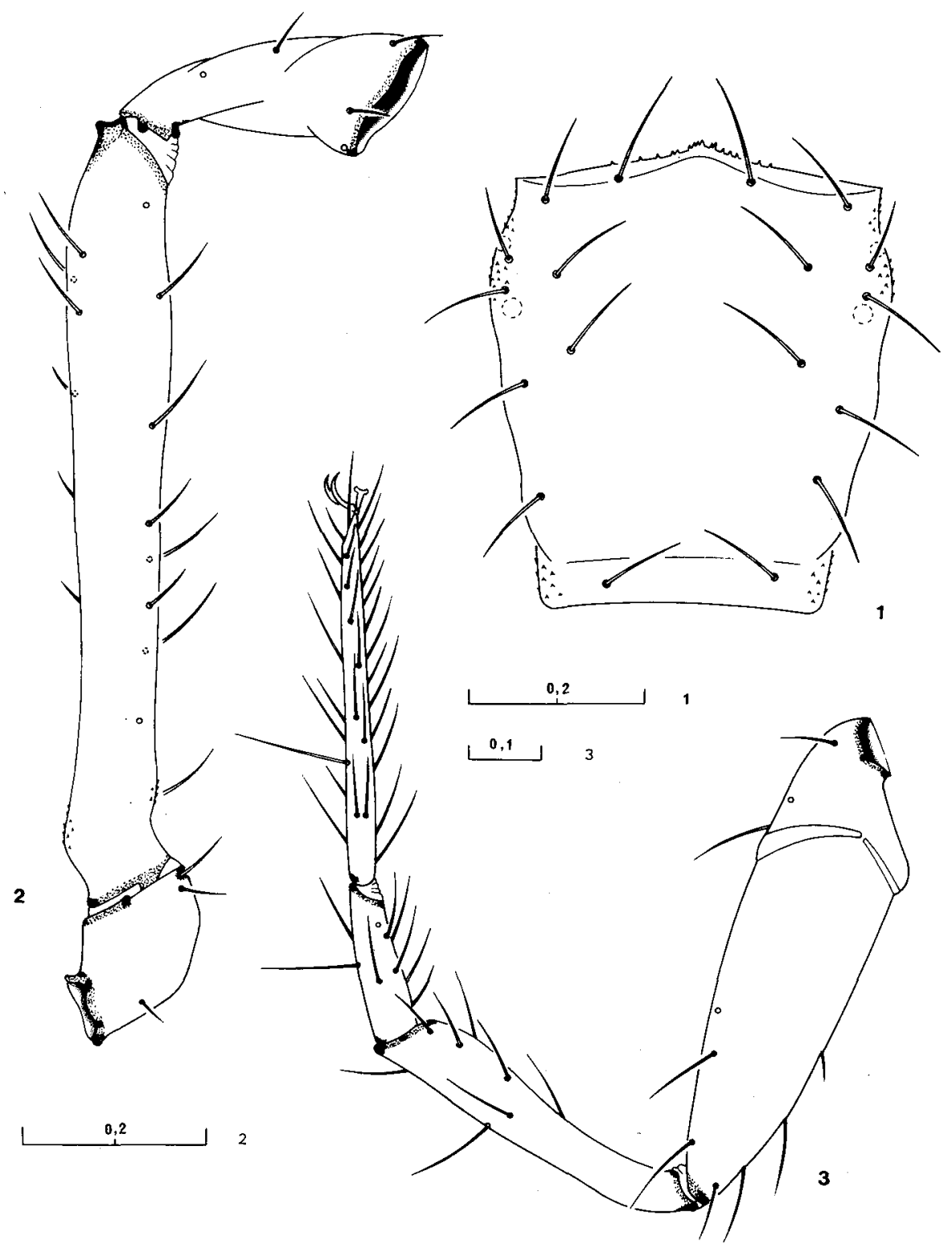

Figs. 1-3.-Chthonius (Ephippiochthonius) verai n. sp., Holotipo. (medidas en mm.): 1.-Carapacho; 2.-Pedipalpo (sin la pinza); 3.-Pata IV. 
Descripción de la $\subsetneq$ Holotipo:

Cuerpo y extremidades en proceso avanzado de despigmentación. Carapacho (Fig. 1) algo más largo que ancho $(1,14 \mathrm{x})$, estrechado hacia las esquinas posteriores, prominente y dentado en el centro del borde anterior; sin ojos, con un par de manchas oculares a cada lado. Quetotaxia: 18 sedas (4-6-4-2-2), sin microsedas preoculares; las sedas supraoculares poco más cortas.

Quetotaxia de los terguitos I-XI: 4-4-4-4-6-6-6-6-6(2 sedas táctiles: ST)-4-6(2 ST), cono anal con dos sedas.

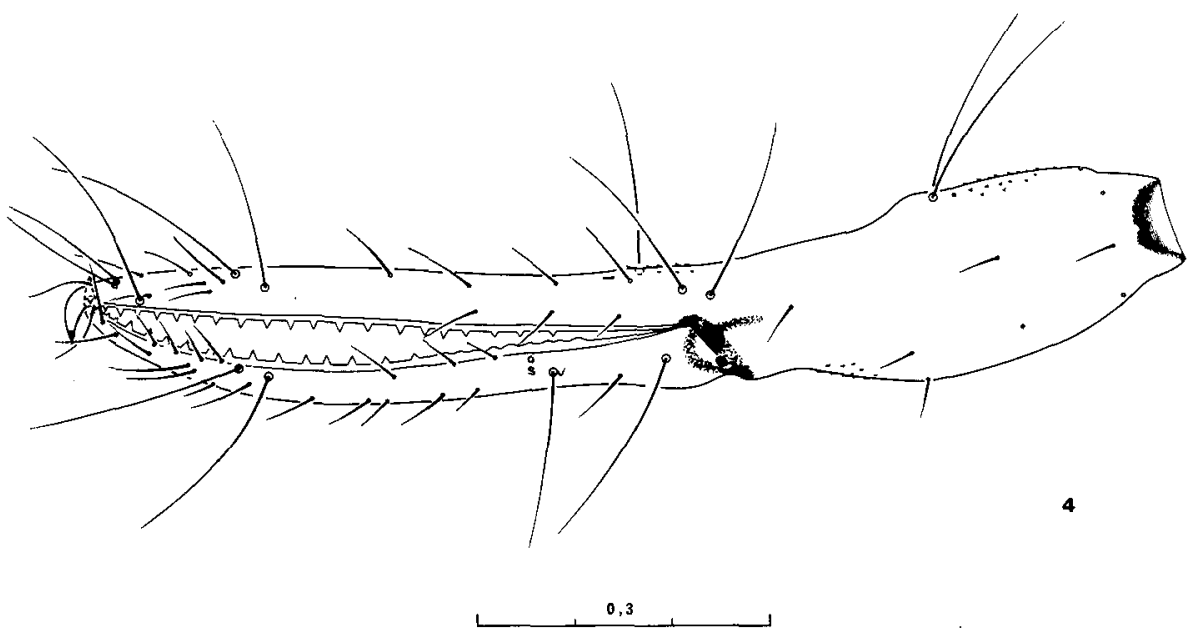

Fig. 4.-Chthonius (Ephippiochthonius) verai n. sp., Holotipo. (medidas en mm.): 4.--Pinza del pedipalpo en visión lateral, s: sensilo.

Quelícero (Fig. 5) con 6 sedas en la mano y una microseda, la seda $v b$ de pequeño tamaño. Serrulla externa con 15 sedas, interna con 13 . Flagelo con 11 sedas, típico del género. Dedo fijo con 2 grandes dientes distales y 9 menores decrecientes en tamaño hasta la base. Dedo móvil con un diente distal grande y 6 menores, sin un diente subapical aislado; seda subgaleal basal del diente distal; el tubérculo sedicígero (Fig. 5a) con forma de un prominente tubérculo propiamente dicho; longitud del dedo móvil: $0,24 \mathrm{~mm}$.

Lóbulo del pedipalpo con 2 sedas, coxa del palpo con 3 sedas, coxa I con 3 sedas y 3 microsedas apicales y marginales, II con 4 sedas y $7 / 11$ espinas coxales: e.c., III con 5 sedas y $6 / 7$ e.c., IV con 6 sedas. Tubérculo intercoxal con 2 sedas. 


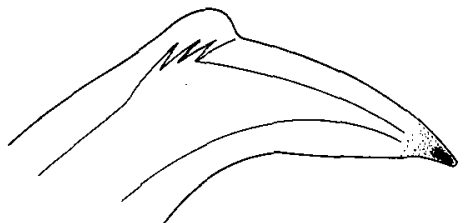

$5 \mathbf{a}$

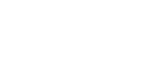

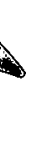
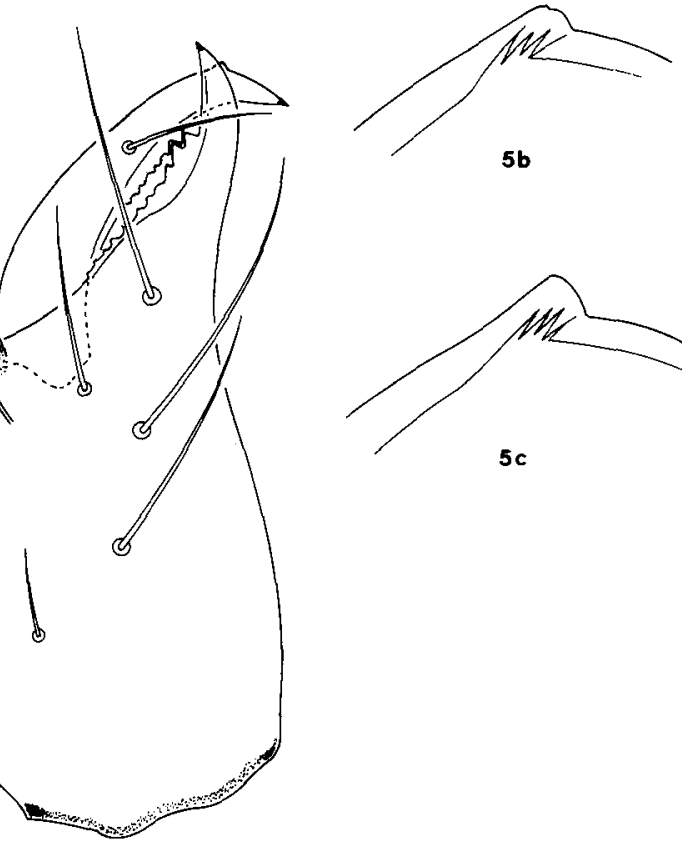

$\mathbf{5 b}$

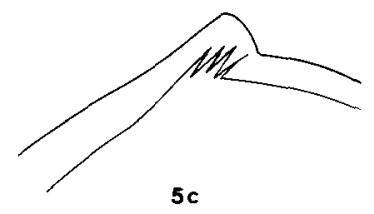

0,2

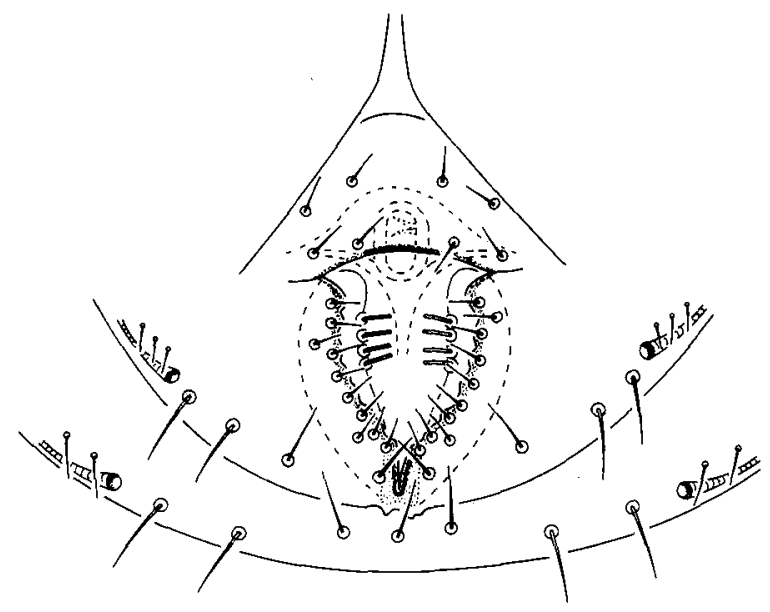

6

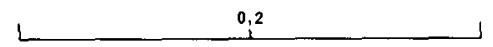

Figs. 5-6.-Chthonius (Ephippiochthonius) verai n. sp., (medidas en mm.). Holotipo: 5.-Quelícero; 5a.-Tubérculo sedicígero del holotipo; 5b.-Id. del alotipo; 5c.-Id. de un paratipo. Alotipo: 6.-Area genital. 
Opérculo genital con 9 sedas. Quetotaxia de los esternitos III-X: 7 sedas y 2 × 3 microsedas estigmales: ms.e.-7 sedas y $2 \times 2$ ms.e. -8 (las sedas laterales: SL diminutas)-6(SL diminutas)-6(SL diminutas)-6(SL cortas)-6-7(2 ST).

Pedipalpo (Figs. 2 y 4): fémur 7,46x más largo que ancho, 2,49x más largo que la tibia, $1,18 \mathrm{x}$ más que el dedo de la pinza y $1,65 \mathrm{x}$ más que el carapacho; tibia, 2,30x. Pinza: $6 \mathrm{x}$; la mano $2,35 \mathrm{x}$; el dedo $1,62 \mathrm{x}$ más largo que da mano; dedo fijo con 21 dientes hasta basal del tricobotrio $a b$, los dos distales y los 4 basales de menor tamaño; dedo móvil con una fila de 15 dientes triangulares hasta poco basal de la mitad de la longitud del dedo (los 2 distales y los 2 basales menores), seguida de una lámina amplia con 9 protuberancias que se extiende hasta basal de $a b$. Tricobotrios: ist claramente distal y separado de esb; et a la altura del $4 .^{\circ}$ diente distal, $t$ basal del $8 .^{\circ}$ diente distal y st distal del $100^{\circ} ; b$ distal de esb y basal de $i s t$; la distancia entre los tricobotrios $s t-a b$ es $2,51 \mathrm{x}$ mayor que la distancia entre $s b-b$. Sensilo presente en la cara externa del dedo, entre la línea de tricobotrios y la dental, poco distal de $s b$.

Pata I: basifémur 6,77x más largo que ancho y 2,0x más largo que el telofémur, éste $3,79 \mathrm{x}$; tibia 5,26x; tarso $12,36 \mathrm{x}$.

Pata IV (Fig. 3): fémur 3,48x; tibia 5,40x; basitarso 3,54x; telotarso $13,13 x$; relación telotarso/basitarso: $2,30 x$. Seda tácil del basitarso cercana a la mitad: 0,49 . ST del telotarsó en el tercio basal: 0,32.

Medidas en $\mathrm{mm}$. :

Longitud del cuerpo: 1,503.

Carapacho: $0,525-0,459$.

Pedipalpo:

Fémur:

$0,865-0.116$

Tibia:

$0,348-0,151$

Mano:

$0,453-0,193$

Dedo:

0,736

Pata I:

Basifémur:

$0,474-0,070$

Telofémur:

$0,235-0,062$

Tibia:

$0,279-0,053$

Tarso:

$0,519-0,042$ 
Pata IV:

Fémur:

$0,699-0,201$

Tibia:

$0,491-0,091$

Basitarso:

$0,223-0,063$

Telotarso:

$0,512-0,039$

Descripción del o* Alotipo:

(Los datos coincidentes con el holotipo son omitidos).

Carapacho $1,11 x$.

Quelícero: serrulla interna con 12 sedas. Dedo móvil con un diente distal grande y 11 dientes más pequeños. Tubérculo sedicígero (Fig. 5b) de tamaño reducido, pero evidente. Longitud del dedo móvil: 0,21 mm.

Coxa II con 4 sedas y 9 e.c., coxa III con 5 sedas y $5 / 6$ e.c.

Opérculo genital con 8 sedas. Abertura genital (Fig. 6) amplia, con 10 sedas sobre cada uno de los bordes, un par de sedas a cada lado algo distantes de la línea de abertura. Cámara genital con $2 \times 4$ «sedas».

Esternito III con 8 sedas y $2 \times 3$ ms.e.

Pedipalpo: fémur 7,37x más largo que ancho y 2,57x más largo que la tibia, $1,23 \mathrm{x}$ más que el dedo de la pinza y $1,69 \mathrm{x}$ más que el carapacho; tibia 2,36x. Pinza: 6,13x; la mano 2,27x; el dedo $1,71 x$ más largo que la mano; dedo fijo con 20 dientes hasta basal de $s b$, los 2 distales menores; dedo móvil con 14 dientes, los 2 distales y los 2 basales pequeños, a continuación una lámina con 13 protuberancias hasta basal de $s b$. La distancia entre $s t-s b$ es $1,90 \mathrm{x}$ mayor que la distancia entre $s b-b$. Sensilo basal y sensiblemente cercano de $s b$.

Pata I: basifémur, 6,97x; telofémur 4,02x; relación basifémur/telofémur: $1,94 \mathrm{x}$; tibia $5,38 \mathrm{x}$; tarso $12,98 \mathrm{x}$.

Pata IV: fémur 3,36x; tibia 5,17x; basitarso $3,63 x$; telotarso $14,25 x$; relación telotarso/basitarso: $2,24 \mathrm{x}$. ST del basitarso: 0,51 , id. del telotarso: 0,33 . 
Medidas en mm.:

Longitud del cuerpo: 1,409.

Carapacho: 0,509-0,460.

Pedipalpo:

Fémur:

Tibia:

Mano:

Dedo:

Pata I:

Basifémur:

Telofémur:

Tibia:

Tarso:

Pata IV:

Fémur:

Tibia:

Basitarso:

Telotarso:
$0,862-0.117$

$0,335-0,142$

$0,410-0,181$

0,700

$0,460-0,066$

$0,237-0,059$

$0,269-0,050$

$0,519-0,040$

Descripción de las $q$ Paratipos:

(Se omiten los datos coincidentes con el holotipo).

Carapacho $1,01 / 1,07 x$. Dos de los paratipos presentan ojos reducidos en el par anterior.

Un paratipo con 3 sedas en el II terguito y otro con 2 ! sedas en el terguito II y 5 ! en el V.

Quelícero: serrula externa con 15/16 sedas. Dedo fijo con 2 grandes dientes distales y $8 / 13$ dientes menores. Dedo móvil con un gran diente distal y $8 / 10$ menores. Tubérculo sedicígero con aspecto de diente (Fig. 5c) o semejante al holotipo. Longitud del dedo móvil: 0,24/ $0,26 \mathrm{~mm}$.

Coxa II con 4 sedas y 12 e.c., III con 5 sedas y $6 / 7$ e.c.

Opérculo genital con $9 / 10$ sedas.

Esternito III con $7 / 8$ sedas y $2 \times 3$ ms.e., IV con $7 / 8$ sedas y $2 \times 2$ ms.e. 
Pedipalpo: fémur 7,12/7,33x más largo que ancho y 2,35/2,47x más largo que la tibia, $1,19 / 1,20 \mathrm{x}$ más que el dedo y $1,65 / 1,76 \mathrm{x}$ más que el carapacho; tibia 2,31/2,40x. Pinza: 5,74/5,93x; la mano $2,19 / 2,37 x$; el dedo 1,53/1,65x más largo que la mano; dedo fijo con $21 / 24$ dientes hasta basal de $s b$, los 2 distales y los $2 / 4$ basales basales son menores; dedo, móvil con una línea de 15/16 dientes, los 2 distales y los 2 basales más peqlieños, seguida de lámina con $9 / 11$ protuberancias hasta basal de $a b$. Tricobotrio $b$ basal de ist o a su misma altura. Distancia entre $s-s b 2,01 / 2,28 \mathrm{x}$ mayor que entre $s b-b$. Sensilo sobre el tricobotrio $s b$ o claramente basal de éste.

Pata I: basifémur $6,85 / 7,01 x$; telofémur 3,50/3,72x; relación basifémur/telofémur: $2,05 / 2,12 \mathrm{x}$; tibia $5,14 / 5,40 \mathrm{x}$; tarso $11,56 / 12,59 \mathrm{x}$.

Pata IV: fémur 3,26/3,46x; tibia 5,19/5,43x; basitarso $3,49 / 3,79 x$; telotarso $13,13 / 13,88 \mathrm{x}$; relación telotarso/basitarso: $2,06 / 2,19 \mathrm{x}$. ST del basitarso: $0,46 / 0,52$, id. del telotarso: 0,30/0,34.

Medidas en mm.:

Longitud del cuerpo: $1,621 / 2,094$.

Carapacho: 0,543/0,579 - 0,539/0,555.

Pedipalpo:

Fémur:

$0,953 / 0,990-0,130 / 0,139$

Tibia:

$0,386 / 0,422-0,161 / 0,183$

Mano:

$0,483 / 0,543-0,221 / 0,229$

Dedo:

$0,797 / 0,833$

Pata I:

Basifémur:

$0,507 / 0,519-0,074 / 0,075$

Telofémur:

$0,241 / 0,253-0,068 / 0,070$

Tibia:

$0,278 / 0,308-0,054 / 0,058$

Tarso:

$0,553 / 0,579-0,046 / 0,048$

Pata IV:

Fémur:

$0,730 / 0,784-0,211 / 0,237$

Tibia:

$0,483 / 0,535-0,089 / 0,103$

Basitarso:

$0,235 / 0,269-0,071 / 0,077$

Telotarso:

$0,555 / 0,591-0,040 / 0,045$

La reducción ocular y la avanzada despigmentación que presenta la especie descrita, unido al hecho de que tan sólo se la ha localizado en los puntos más profundos de la cavidad, hacen pensaț que pudiera tratarse de una especie troglófila o neotroglobia, sin poder descartar que pudiera hallársela al exterior. 
Derivatio nominis: Dedicada por el autor a la memoria de su buen amigo y compañero de múltiples exploraciones espeleológicas: José Manuel Vera Catral, fallecido en 1976 en el Complejo Hundidero-Gato (Málaga). José Manuel intuyó acertadamente la importancia biogeográfica de las recolecciones faunísticas en la Cova del Tío Melxor e inició sucesivas campañas de recolección, fruto de aquellas fue la captura del catópido cavernícola Spelaeochlamys verai, la especie más meridional del género.

\section{DISCUSION}

De entre las especies epigeas ibéricas con lámina basal en el dedo móvil de la pinza del pedipalpo, Chthonius (E.) verai $\mathrm{N}$. sp. se distingue de C. machadoi VACHON (1940) (O. Península Ibérica, Islas Canarias, Marruecos) y de C. pyrenaicus BEIER (1934) (Pirineos, Cataluña, Baleares) por la ausencia de un diente subapical aislado en el dedo móvil del quelícero y por un mayor tamaño de los miembros, de C. asturiensis BEIER (1955) (N. España) por la posición de los tricobotrios $i s t-e s b-e b$, las proporciones del pedipalpo y el número de dientes sobre el dedo móvil de la pinza.

La nueva especie presenta ciertas semejanzas con especies primordialmente mediterráneas con hábitos cavernícolas y ojos reducidos o ausentes; no obstante, se separa fácilmente de ellas: de $C$. cassolai BEIER (1973) (Cèrdeña) por la quetotaxia del borde delantero y trasero del carapacho, el número de tubérculos de la lámina basal del dedo de la pinza y la relación dedo/mano del pedipalpo. De $C$. siscoensis HEURTAULT (1975) (Córcega) por la quetotaxia de las microsedas del carapacho y del quelícero, la relación dedo/mano del pedipalpo, el número de dientes sobre el dedo móvil de la pinza, la posición del tricobotrio $b$ respecto a ist, etc. De C. minutus VACHON (1940) (Portugal) y de C. corcyraeus MAHNERT (1976) (Korfu) como distinción fundamental por la ausencia de diente subapical en el dedo móvil del quelícero.

Más cercana a otras especies cavernícolas levantinas, como C. pinai ZARAGOZA (1985), C. sendrai ZARAGOZA (1985) y C. ventallo $i$ BEIER (1939). Fácilmente separable de $C$. pinai por la quetotaxia de las microsedas en el carapacho y en el quelícero y por las medidas del pedipalpo; igualmente de $C$. sendrai por la presencia en la especie valenciana de un diente subapical en el dedo móvil del quelícero. Mucho más sutiles son las diferencias con $C$. ventalloi, de la que se distingue por: la ausencia de ojos y total despigmentación en $C$. ventalloi; las medidas del pedipalpo, mayores en la nueva especie; la distancia entre los 
tricobotrios $s t-s / s b-b ;$ y un buen número de detalles menores no menos importantes: la posición de la seda táctil en el basitarso de la pata IV, cercana a la mitad en $C$. verai y alejadamente basal en $C$. ventalloi; el tubérculo sedicígero claramente mayor en ambos sexos de la nueva especie y también, una cierta variación en la posición del sensilo sobre el dedo móvil de la pinza, etc. 


\section{BIBLIOGRAFIA}

BEIER, M., (1934). Neue cavernicole und subterrane Pseudoscorpione. Mtt. Höhlen, u. Karst., $53-59$.

BEIER, M., (1939). Die Pseudoscorpioniden-Fauna der Iberischen Halbinsel. Zool. Jahr., Band 72, $1 / 2: 157-202$.

BEIER, M., (1955). Neue Beiträge zur Kenntnis der Iberischen Pseudoscorpioniden-Fauna, Eos, 31: $87-122$.

BEIER, M., (1973). Neue Funder von Höhlen-Pseudoskorpionen auf Sardinien. Ann. Natur. Mus. Wien, 77: 163-166.

HEURTAULT, J., (1975). Deux nouvelles espèces de pseudoscorpions Chthoniidae (Arachnides) cavernicoles de Corse: Chthonius (E.) remyi et Chthonius (E.) siscoensis. Ann. Spéléol., 30, 2-313-318.

MAHNERT, V., (1976). Zwei neue Pseudoskorpion-Arten (Arachnida) aus griechischen Höhlen (Über griechische Pseudoskorpione VII). Ber. nat. med. Ver. Innsbruck, Band 63: 177-183.

VACHON, M., (1940). Éléments de la Faune Portugaise des Pseudoscorpions (Arachnides) avec description de quatre espèces nouvelles. Pub. Inst. zool. Fac. Ci. Porto, 2: 7-30.

ZARAGOZA, J.A., (1985) (en prensa). Nuevos o interesantes Chthoniidae cavernícolas del País Valenciano (Arachnida, Pseudoscorpiones). Misc. Zool., IX. 